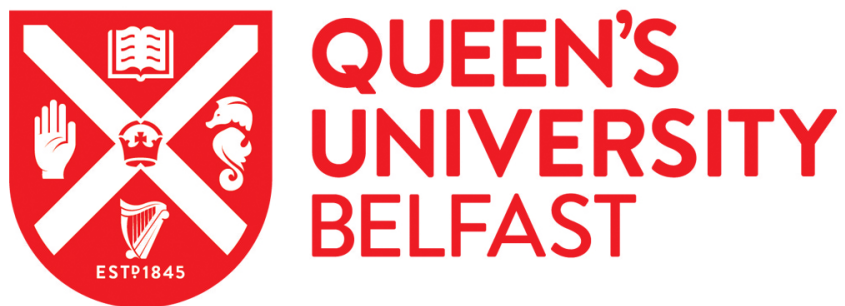

\section{Anomalous temperature dependence of layer spacing of de Vries liquid crystals: Compensation model}

Merkel, K., Kocot, A., Vij, J. K., Stevenson, P. J., Panov, A., \& Rodriguez, D. (2016). Anomalous temperature dependence of layer spacing of de Vries liquid crystals: Compensation model. Applied Physics Letters, 108(24), [243301]. https://doi.org/10.1063/1.4953598

Published in:

Applied Physics Letters

Document Version:

Publisher's PDF, also known as Version of record

Queen's University Belfast - Research Portal:

Link to publication record in Queen's University Belfast Research Portal

Publisher rights

Copyright 2016 AIP Publishing. This work is made available online in accordance with the publisher's policies. Please refer to any applicable terms of use of the publisher.

\section{General rights}

Copyright for the publications made accessible via the Queen's University Belfast Research Portal is retained by the author(s) and / or other copyright owners and it is a condition of accessing these publications that users recognise and abide by the legal requirements associated with these rights.

Take down policy

The Research Portal is Queen's institutional repository that provides access to Queen's research output. Every effort has been made to ensure that content in the Research Portal does not infringe any person's rights, or applicable UK laws. If you discover content in the Research Portal that you believe breaches copyright or violates any law, please contact openaccess@qub.ac.uk. 


\section{Anomalous temperature dependence of layer spacing of de Vries liquid crystals: Compensation model}

K. Merkel, A. Kocot, J. K. Vij, P. J. Stevenson, A. Panov, and D. Rodriguez

Citation: Appl. Phys. Lett. 108, 243301 (2016); doi: 10.1063/1.4953598

View online: https://doi.org/10.1063/1.4953598

View Table of Contents: http://aip.scitation.org/toc/apl/108/24

Published by the American Institute of Physics

\section{Articles you may be interested in}

The description of the smectic $A$ and $C$ phases and the smectic $A-C$ phase transition of TCOOB with a diffusecone model

The Journal of Chemical Physics 71, 25 (1979); 10.1063/1.438123

Observation of the de Vries behavior in $\mathrm{SmA}^{*}$ phase of a liquid crystal using polarised Raman scattering and infrared spectroscopy

The Journal of Chemical Physics 147, 094903 (2017); 10.1063/1.4999792

Submicrosecond bistable electro-optic switching in liquid crystals

Applied Physics Letters 36, 899 (1980); 10.1063/1.91359

Wide range room temperature electroclinic liquid crystal mixture with large induced tilt and very small layer contraction

Journal of Applied Physics 119, 124103 (2016); 10.1063/1.4944607

Orientational distribution functions and order parameters in "de Vries"-type smectics: A simulation study

The Journal of Chemical Physics 145, 134901 (2016); 10.1063/1.4963660

Electro-optic characteristics of de Vries tilted smectic liquid crystals: Analog behavior in the smectic $A^{*}$ and smectic $C^{*}$ phases

Applied Physics Letters 80, 4097 (2002); 10.1063/1.1480472

\section{HDDEN}

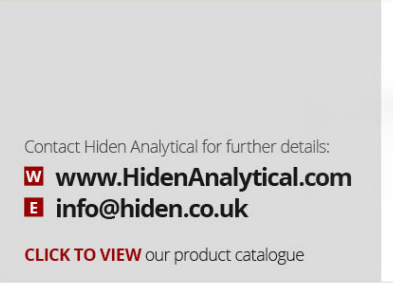

\section{Instruments for Advanced Science}
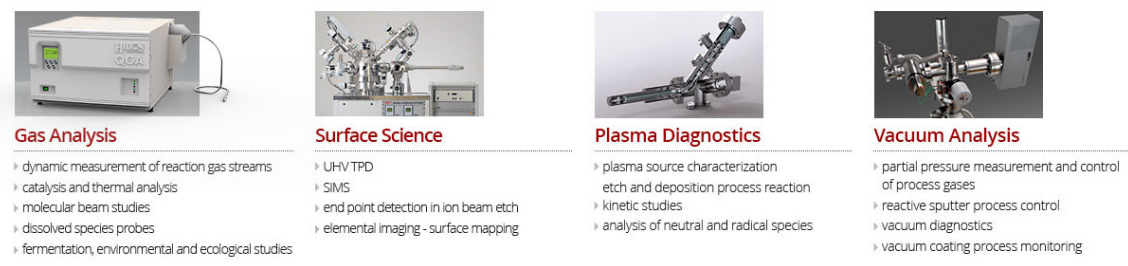


\title{
Anomalous temperature dependence of layer spacing of de Vries liquid crystals: Compensation model
}

\author{
K. Merkel, ${ }^{1}$ A. Kocot, ${ }^{2}$ J. K. Vij, ${ }^{3, a)}$ P. J. Stevenson, ${ }^{4}$ A. Panov, ${ }^{4}$ and D. Rodriguez ${ }^{4}$ \\ ${ }^{1}$ Central Mining Institute, Katowice 40-166, Poland \\ ${ }^{2}$ Institute of Physics, Silesian University, Katowice 40-007, Poland \\ ${ }^{3}$ Department of Electronic and Electrical Engineering, Trinity College, The University of Dublin, \\ Dublin 2, Ireland \\ ${ }^{4}$ School of Chemistry and Chemical Engineering, Queens University Belfast, Belfast BT7 INN, \\ Northern Ireland, United Kingdom
}

(Received 23 March 2016; accepted 27 May 2016; published online 13 June 2016)

\begin{abstract}
Smectic liquid crystals that exhibit temperature independent layer thickness offer technological advantages for their use in displays and photonic devices. The dependence of the layer spacing in $\mathrm{SmA}$ and SmC phases of de Vries liquid crystals is found to exhibit distinct features. On entering the $\mathrm{SmC}$ phase, the layer thickness initially decreases below $\mathrm{SmA}$ to $\mathrm{SmC}\left(\mathrm{T}_{A-C}\right)$ transition temperature but increases anomalously with reducing temperature despite the molecular tilt increasing. This anomalous observation is being explained quantitatively. Results of IR spectroscopy show that layer shrinkage is caused by tilt of the mesogen's rigid core, whereas the expansion is caused by the chains getting more ordered with reducing temperature. This mutual compensation arising from molecular fragments contributing to the layer thickness differs from the previous models. The orientational order parameter of the rigid core of the mesogen provides direct evidence for de Vries cone model in the SmA phase for the two compounds investigated. Published by AIP Publishing.

[http://dx.doi.org/10.1063/1.4953598]
\end{abstract}

Self-organization of the amphiphilic molecules forming liquid crystal phases is believed to occur through nanosegregation of two or more incompatible segments into distinct domains where topographies are dictated by the molecular shape and the amphiphilic interfacial curvature. ${ }^{1}$ Formation of the lamellar smectic phases by calamitic (rod-shaped) mesogens is driven by nano-segregation of rigid cores, flexible aliphatic side chains, and by incompatible molecular segments such as the fluorinated side-chains or oligomeric siloxanes. The fluid smectic A ( SmA) and smectic C (SmC) phases have diffused lamellar structures described by a density wave with a period $d$ corresponding to the smectic layer spacing. ${ }^{2}$ In the uniaxial SmA phase, the axes of the orientational and translational orders coincide with each other in which the director $n$ is parallel to the smectic layer normal $z$. In the biaxial SmC phase, $n$ is tilted relative to $z$ by the tilt angle $\theta$ that varies with temperature. According to the classic rigid-rod model, SmA-SmC phase transition is accompanied by a contraction of the layer spacing $d$ that scales with the cosine of the tilt angle $\theta$. The layer contraction in conventional calamitics is as large as $13 \% .{ }^{1}$ In a separate class of liquid crystals known as de Vries smectics, the layer contraction is only of the order of a fraction of $1 \%$ or less, but it behaves anomalously in the $\mathrm{SmC}$ phase: instead of decreasing, it increases with a reduction in temperature. ${ }^{3-5}$

The de Vries model ${ }^{6}$ assumes that molecules are titled but azimuthally distributed on a cone in the SmA phase. The degeneracy in azimuthal distribution breaks down at the $\mathrm{SmA}$ to SmC transition where it condenses towards a preferred value but without a change in the tilt angle. The layer shrinkage as a consequence is absent close to the transition

a)jvij@tcd.ie temperature. However, this model cannot explain as to why the layer thickness in SmC phase anomalously increases with the reduction in temperature unless an additional feature is introduced. Some of the current molecular models ${ }^{7,8}$ therefore assume that the orientational order parameter in SmA is low and it increases in SmC phase, thereby increasing $d(T)$ through reduced molecular fluctuations with a reduction in temperature. A contraction in $d(T)$ is caused by increasing tilt and expansion by increase in the orientational order parameter with decreasing temperature. However in many cases, this order parameter is already found to be saturated and its increase with a reduction in temperature is insignificant. In a model given by Yoon et al., ${ }^{4}$ the main contribution to the compensation in the layer expansion comes from increase in the effective molecular length, $L(T)$ with a reduction in temperature (see Figure 7 in Ref. 4). They initially used the formula derived by Lagerwall et al. ${ }^{9} L(T)$ $=\frac{3 d(T)}{(S+2) \cos \alpha(T)}$, where $\alpha(T)$ and $d(T)$ are the temperature dependent tilt angle and layer thickness, respectively. Both $L(\mathrm{~T})$ and $S$ of the hydrocarbons (which included the rigid core) were varied to explain the observed anomalous temperature dependence of $d(T)$. S for hydrocarbons was already large in the SmA phase $(\sim 0.75$ for $\mathrm{C} 4$ and $\sim 0.7$ for $C 9)$ and almost temperature independent. Hence, layer shrinkage could hardly be compensated by a further increase in $S$. They obtained $L(T) \approx 50 \AA$ for both $\mathrm{C} 4$ and $\mathrm{C} 9$. This length is much greater than the maximal extended lengths of $\mathrm{C} 4$ and C9 calculated from density functional theory (DFT) (39 and $45 \AA$, respectively). However, if we were to add another siloxane group to the length of the molecule, then it may be different. Based on their experimental results ${ }^{10}$ of $\left\langle P_{2}\right\rangle,\left\langle P_{4}\right\rangle$, and $\left\langle P_{6}\right\rangle$ for the four compounds, including $\mathrm{C} 4$ and $\mathrm{C}$, they 
calculated the orientational distribution function $f(\theta)$ and found this narrowed considerably in going from SmA to the $\mathrm{SmC}$ phase. They also modified the layer thickness equation to $d(T)=L\langle\cos \theta\rangle \cos \alpha(T), L$ is the effective length of the molecule, $\langle\cos \theta\rangle=\frac{S+2}{3}$ represents the average molecular fluctuations about the director $\mathbf{n}$; these are reflected in $f(\theta)$. This implies that $L$ is even longer in this case.

By using the polarized IR, one can however separate out the orientational order parameters of the hydrocarbons ${ }^{4,10}$ in terms of the aliphatic chains and the rigid core and find the order parameter of the siloxane group. We can thus examine the role played by the chains in giving rise to anomalous temperature dependence of $d(T)$ in the compensation model, where the contribution from one fragment increases and second decreases and expansion in the length of one fragment overtakes the contraction in the second leading to anomalous expansion in $d(T)$ with a reduction in temperature. On finding out exactly as to how each fragment contributes to the layer thickness, compounds for the next generation of displays can be successfully designed. The structure of compounds C4/C9 is given in Fig. 1(a). The molecule depicted on de Vries cone in Fig. 1(b) is subdivided into four segments (see Fig. 1(c)): the rigid core (yellow), a trisiloxane (TS) group (blue), and two aliphatic chains (orange): spacer $\mathrm{C}_{11} \mathrm{H}_{22}$ and the tail $\mathrm{C}_{\mathrm{n}} \mathrm{H}_{2 \mathrm{n}+1}$, on opposite sides of the rigid core. The trisiloxane (TS) tail where silicon atoms preferentially orient in staggered conformation (Fig. 1(d)) is bulkier than others and drives nano-segregation. The lateral intermolecular distance between the neighboring molecules is determined by a cross-section of the fatty parts of the molecular tail (cross section diameter is $6.5 \AA$ ). On cooling the sample, the chain ordering increases and this allows for an expansion of the tightly packed segments. X-Ray scattering studies $^{4}$ do confirm nano-segregation of the siloxanes from hydrocarbons (includes the rigid core). The lateral separation of the groups is almost equal to its cross-sectional diameter such that TS group cannot overlap hydrocarbons. This leads to staggered arrangement of molecules shown in Fig. 1(d). The arrangement in layers is dictated by the molecular head and tail equivalence in SmA and SmC phases. Trisoloxanes tails occupy the space with a cross sectional diameter of $(6.8 \AA)^{2}=46.0 \AA$, two times larger than the cross sectional diameter of the hydrocarbons, 2. $(4.5 \AA)^{2}=40.5 \AA^{2}$, where $6.8 \AA$ and $4.5 \AA$ are the wide angle peak positions observed for the siloxanes and hydrocarbons. ${ }^{4}$ According to this model, alkyl chains have more orientational freedom than the TS group and the rigid core. The tilt angle of the chains, on average, may not change, but the chains get extended with reducing temperature this is reflected in the order parameter.

For aligning the sample homeotropically, both inside surfaces of $\mathrm{ZnSe}$ windows were coated with carboxylato chromium complex' chromolane; this is cured at a temperature of $150{ }^{\circ} \mathrm{C}$. In the homeotropic configuration, absorbance measurements are made in two orthogonal directions in SmA and SmC phases. $A_{X X}$ and $A_{Y Y}$ in this case are found to be approximately equal. We use the following equation for

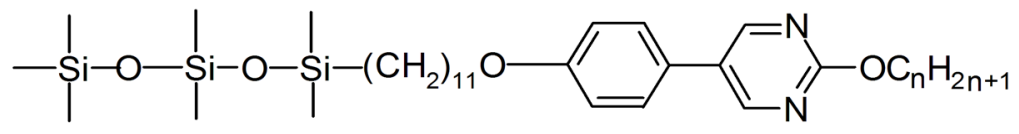

(b)

$$
\begin{aligned}
& \mathrm{n}=4: \mathrm{C} 4: \mathrm{Cr} \stackrel{35^{\circ} \mathrm{C}}{\longleftarrow} \mathrm{SmC} \stackrel{57^{\circ} \mathrm{C}}{\longleftarrow} \mathrm{SmA} \stackrel{79^{\circ} \mathrm{C}}{\longleftarrow} \mathrm{I} \\
& \mathrm{n}=9: \mathrm{C} 9: \mathrm{Cr} \stackrel{51^{\circ} \mathrm{C}}{\longleftarrow} \mathrm{SmC} \stackrel{73^{\circ} \mathrm{C}}{\longleftarrow} \mathrm{SmA} \stackrel{76^{\circ} \mathrm{C}}{=}
\end{aligned}
$$

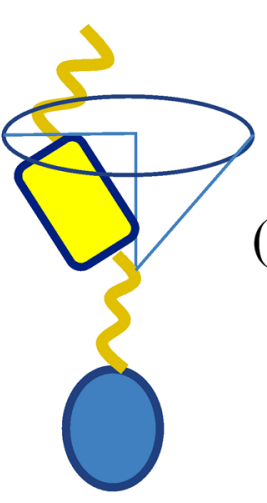

(c)

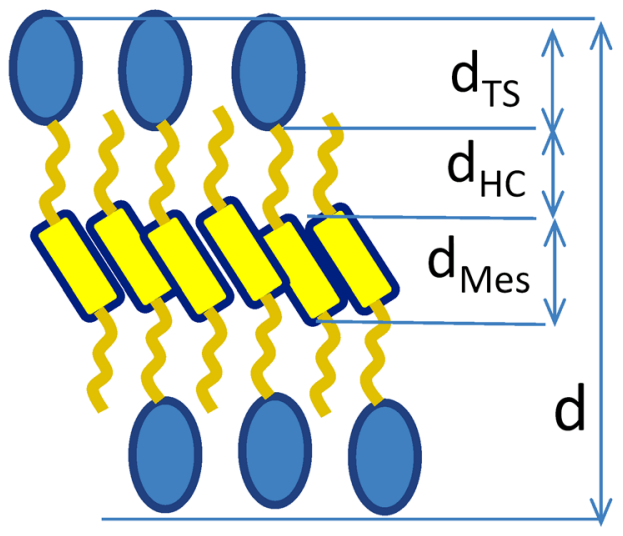

(d)

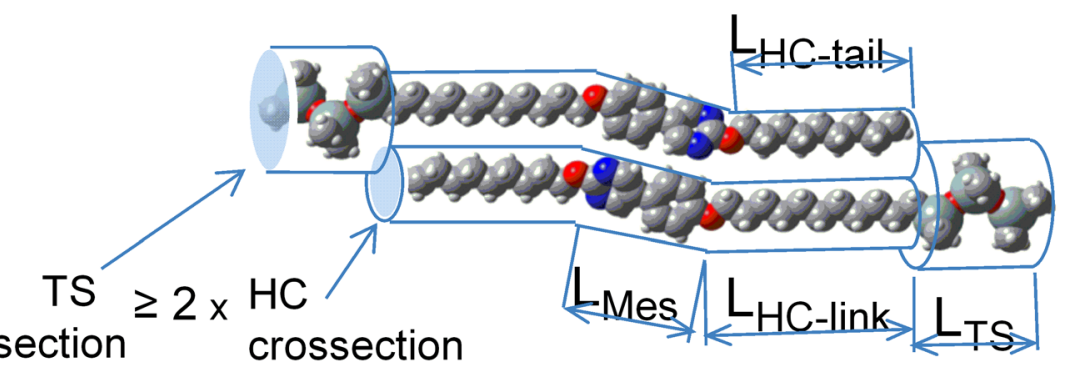

FIG. 1. (a) Molecular structure and phase sequences of $\mathrm{C} 4$ and $\mathrm{C} 9$, synthesis given in the supplementary material, ${ }^{11}$ (b) molecule on de Vries cone, and (c) arrangement of molecules in a smectic layer (d). C9 is divided in four segments, head and tail equivalence of molecules in the layer, the alkoxy chains are in all trans-conformation; length of the mesogen's core, $L_{\mathrm{Mes}}$, includes oxygen atoms on both sides. 
calculating $S$ and $D$ of the transition dipole moments of a vibrational band: ${ }^{12}$

$$
\begin{aligned}
\left(A_{X X}+A_{Y Y}\right) / 2 A_{0}= & 1+S\left(\frac{3}{2} \sin ^{2} \beta-1\right) \\
& +\frac{1}{2} D\left(\sin ^{2} \beta \cos 2 \gamma\right) .
\end{aligned}
$$

$\beta$ is the polar angle between the transition dipole moment and the molecular $z$-axis, $\gamma$ is the azimuthal angle, the angle between the projection of the transition dipole moment on the $x-y$ plane whereas both $x$ and y axes correspond to the molecular frame of reference. $S$ and $D$ are the Saupe order parameters $S_{i, j}{ }^{12,15}$ in the laboratory frame, and the $Z$ axis coincides with smectic layer normal. $S=S_{z z} ; D=S_{x x}-S_{y y}$. Ao is the sum of the three absorbances' components measured along the three orthogonal axes in the laboratory frame in the isotropic phase. The dominant vibrational bands are determined from the molecular structure. For the rigid core, the phenylpyrimidine stretching vibrations centred at $\sim 1160$ or $\sim 1598 \mathrm{~cm}^{-1}$ are chosen; these act along the phenyl para axis of the molecule. The pyrimidine asymmetric stretching band centred at $\sim 1547 \mathrm{~cm}^{-1}$ is additionally chosen. If $\beta \approx 0^{\circ}$ then Eq. (1) shows, $\gamma$ needs not be known. In many cases, the molecular calculations for a vibrational band of interest are used in finding $\beta$. For $1547 \mathrm{~cm}^{-1}, \beta$ is found as $\approx 70^{\circ}$. It is convenient to set the $x$ axis in the pyrimidine plane, so $\gamma=0$ for the band. $S$ of the rigid core calculated for phenyl para axis is shown in Fig. 2. $S$ initially increases on decreasing temperature in SmA phase, followed by its decrease on transition to the $\mathrm{SmC}$ phase due to increased tilt. This is a consequence of its definition by Saupe. ${ }^{15}$ At the SmA-SmC transition, a decrease in $S$ is discontinuous for $\mathrm{C} 9$, confirming its first-order character, but it is continuous in $\mathrm{C} 4$. The molecular biaxiality parameter $D$ for the rigid core is significantly large in the SmA phase $(0.38$ for $\mathrm{C} 4$ and 0.34 for $\mathrm{C} 9$ ), it gradually increases on cooling in $\mathrm{SmC}$ where at low temperatures it approaches $D=0.6$. The increase in $D$ influences the dependence of the distribution function on its azimuthal angle in the molecular frame of reference. $f(\theta)$ gets narrower in $\mathrm{SmC}$ compared to $\mathrm{SmA}$, results in line with that found using $\mathrm{X}$-rays. ${ }^{10}$ The two results though qualitatively similar cannot be quantitatively compared. In the

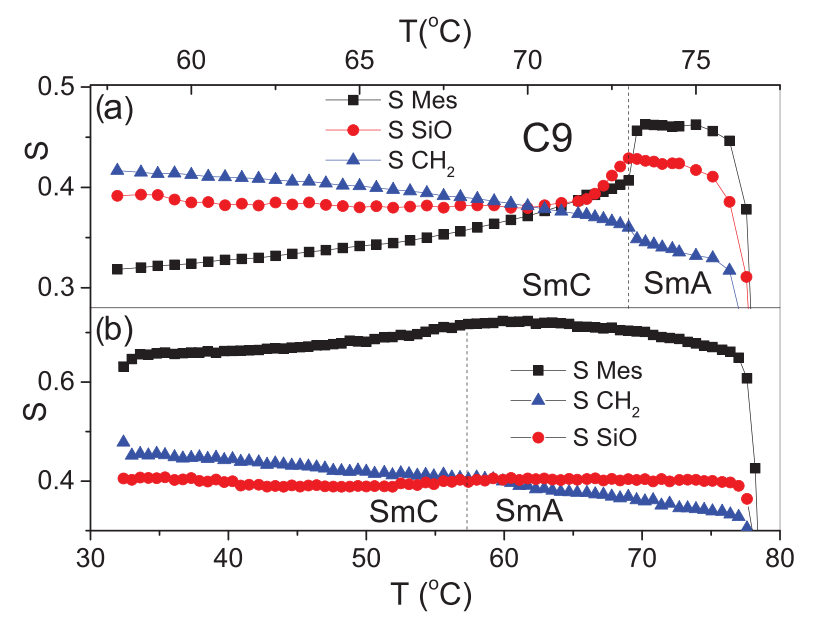

FIG. 2. The orientational order parameters (a) C9 and (b) C4 calculated for - - mesogen's rigid core, red $-\mathrm{TS}$, and blue $\mathbf{\Delta}-\mathrm{CH}_{2}$ groups. formalism of Volkenstein and Flory, ${ }^{16,17}$ the chain itself is expected to have conformational flexibility associated with the internal degrees of freedom of rotations around skeleton bonds in the tail. The reference axis of the chain segment is chosen perpendicular to the $\mathrm{HCH}$ plane. For a perfectly oriented chain in "all-trans" conformation, the segment axis is parallel to the main axis of the chain, while all $\mathrm{CH}_{2}$ transition dipoles are perpendicular to this axis. The order parameter defined for each $\mathrm{CH}_{2}$ segment is expected to be dependent on its position in the chain (how far a methylene group is situated from the core). However using IR absorbances, we can only calculate the average order parameter of the aliphatic chain by using symmetric and asymmetric stretching bands of the $\mathrm{CH}_{2}$ group, centred at $2854 \mathrm{~cm}^{-1}$ and $2924 \mathrm{~cm}^{-1}$. Similarly, the reference axis of the trisiloxane tail segment- $\mathrm{Si}\left(\mathrm{CH}_{3}\right)_{2}$ is defined as normal to the $\mathrm{C}-\mathrm{Si}$-C plane. $\mathrm{S}$ for TS is calculated using absorbances for stretching band of $\mathrm{Si}\left(\mathrm{CH}_{3}\right)_{2}$ group, at $1257 \mathrm{~cm}^{-1}$. $S$ for different segments for both $\mathrm{C} 4$ and $\mathrm{C} 9$ are plotted in Fig. 2.

$S$ for the rigid core of $\mathrm{C} 4$ is higher than for $\mathrm{C} 9$ because of the lower tilt in C4. $S$ for the aliphatic chains increases and it contrasts with the rigid core, and behaves similarly in both $\mathrm{SmC}$ and SmA phases. $S$ of the trisiloxane (TS) group is lower $(\sim 0.4$ in $\mathrm{SmA})$ than the rigid core while it is weakly temperature dependent. This means that TS is less ordered, and due to flexibility; its tilt from the layer normal is almost zero, in qualitative agreement with X-ray scattering ${ }^{4}$ result. The chains are disordered and are situated on both sides of the rigid core and we therefore expect their tilt to be zero. $D$ for chains is found to be zero. A continued increase is $S$ may only imply that a projection of the length of the chain segments on the layer normal is increasing while all methylene links are getting in trans configuration with temperature reducing. Hence, the model where the molecule is divided into more moieties gives better information compared to the case when its orientation is considered as an entity. X-rays can determine up to three even order parameters of the Legendre polynomial from where a distribution function for a uniaxial system can be constructed. ${ }^{4}$ The IR studies lead to the biaxial $D$ parameter that gives information about the molecular biaxiality in the distribution function. $D$ varies from 0.3 to 0.6 for $\mathrm{C} 4$ and 0.4 to 0.6 for C9.

The optical anisotropy of liquid crystals is determined mostly by the orientational order parameter of the rigid core. In the SmC phase, the molecules on the average are tilted and their long axes have a narrower azimuthal distribution as stated before. The order parameter of the mesogen is related to average $\left\langle\cos ^{2} \theta_{\text {mes }}\right\rangle=(2 S+1) / 3$, where $\theta_{\text {mes }}$ is considered as the cone angle for the rotation of the rigid core. Fig. 3 shows that cone angle of the rigid core gets larger on transition to the $\mathrm{SmC}\left(36.7^{\circ} \rightarrow 42.2^{\circ}\right.$ for $\mathrm{C} 9$ and $25.4^{\circ} \rightarrow 28.6^{\circ}$ for $\mathrm{C} 4$ ). Since the azimuthal distribution is degenerated in the SmA phase, the optical axis therefore lies along the layer normal. Thus, the optical tilt angle $\theta_{\text {opt }} \cong 0$ and the molecular tilt $\theta_{\text {mes }}$ is large. On entering $\mathrm{SmC}$, the azimuthal distribution becomes narrower as the azimuthal degeneracy is broken. $\theta_{\text {opt }}$ therefore becomes finite and finally approaches the cone angle, $\theta_{\text {mes }}$, for the rigid core (see Fig. 3). A similar increase in $\theta_{\text {mes }}$ is induced by electric field in SmA. This is shown by the electric field dependence of birefringence and $\theta_{\text {opt }}{ }^{13,14,18}$ 


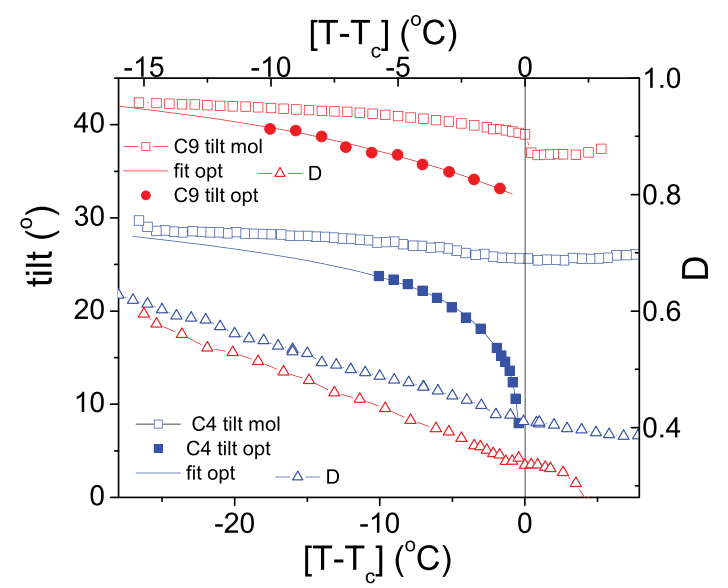

FIG. 3. Optical and molecular tilts. $D$ for the rigid cores (of C4 and C9) calculated from absorbances for $1597 \mathrm{~cm}^{-1}$. Scale of $D$ is fixed on the right hand column. Data for the optical tilt are taken from Ref. 5.

The characteristic features of the temperature dependencies of the layer spacing seen in Figure 4, for both $\mathrm{C} 4$ and $\mathrm{C} 9,{ }^{4,5}$ are quantitatively explained here. On entering $\mathrm{SmC}$, the layer thickness initially decreases and then starts to increase while the molecular tilt angle is still growing, one would have normally expected thickness to continually decrease with reducing temperature. Origin of such anomalies lies in different orientational behaviour of different molecular parts, i.e., rigid core, the aliphatic chain, and the trisiloxane tail groups. The model is based on considering the layer thickness as a sum of the contributions from each of the molecular segments (index $i$ ), represented by an averaged projection of its length $L_{i}$ along the layer normal $d=\sum_{i} L_{i}\left\langle\cos \theta_{i}\right\rangle$, and $\theta_{i}$ is the angle between the $i$ th segment axis and the layer normal. We can thus calculate contributions from all molecular segments to the total layer thickness from its order parameter dependent on $\left\langle\cos ^{2} \theta\right\rangle$. If the local fluctuation of the director is not large, i.e., $S \geq 0.4$, we can approximate $\langle|\cos \theta|\rangle \cong\left\langle\cos ^{2} \theta\right\rangle^{1 / 2}$. On assuming the Maier-Saupe distribution function, ${ }^{15}$ for $S=0.4, \cos (\theta)$ calculated as $\left\langle\cos ^{2} \theta\right\rangle^{1 / 2}$ is overestimated by $5 \%$, while it is reasonably accurate for higher values of $S$. For $S=0.7$, the overestimation of $\cos \theta$ is approximately $1 \%$. The model assumes a

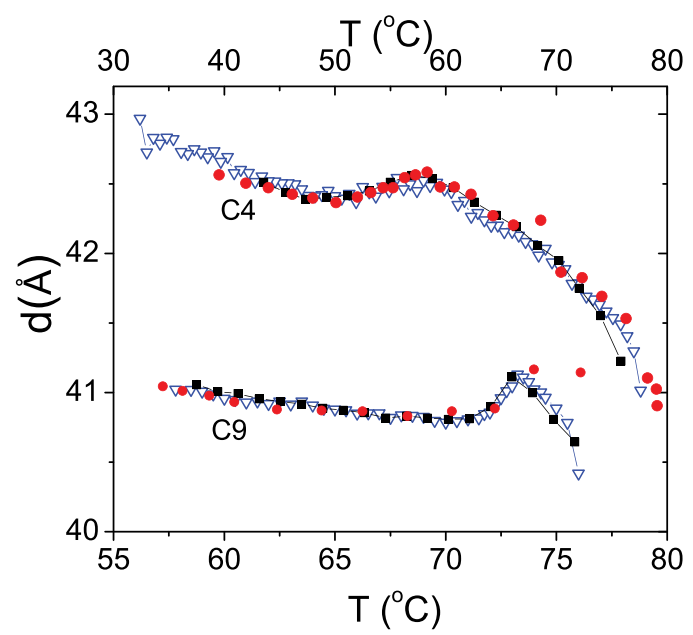

FIG. 4. Temperature dependencies of the layer thicknesses for $\mathrm{C} 4$ and $\mathrm{C} 9$; data red - - Ref. 4, and $\square$-Ref. 5 scaled to values in Ref. 4 at $T=T_{c}$, blue $\nabla$ - the current model. decoupling of the aliphatic chains from the rigid core and the trisiloxane tail. On recalling the temperature dependence of the order parameter shown in Fig. 2, it is quite clear that contribution of the hard core to $d$ decreases at the transition $T_{A C}$ but that of the aliphatic chain increases. These contributions initially compensate each other close to the transition temperature, but at lower temperatures, contribution of the chain to the layer thickness dominates over the rigid core, leading to an increase in the layer thickness.

C9 in all trans conformation is subdivided into four parts, as illustrated in Fig. 2, the rigid core of the length $L_{M e s}=9.8 \AA$, TS tail group, $L_{T S}=8.8 \AA$, and two aliphatic groups one as a spacer $\mathrm{C}_{11} \mathrm{H}_{22}, L_{\text {HClink }}=14 \AA$, and second $\mathrm{C}_{\mathrm{n}} \mathrm{H}_{2 \mathrm{n}+1}$ as a aliphatic tail, $L_{\text {HCtail }}=13.2 \AA$. Figure $1(\mathrm{~d})$ defines the length of each segment, $L_{\mathrm{i}}\left(=1.27 \AA\right.$ for $\left.-\mathrm{CH}_{2}\right)$, where $L_{\mathrm{i}}=L / n$, where $L$ is the total length of the chain in all-trans conformation, and $n$ is the number of its segments. Each segment makes different tilt with the layer normal (positive or negative); thus, the average tilt is most likely to be zero, but it is still possible to determine the average projection of each segment on the layer normal from its order parameter. Thus, the contribution of each segment to the layer thickness is a product of its length and $\left\langle\left|\cos \theta_{i}\right|\right\rangle$. Thus, $d$ is found as an additive sum of contributions arising from different segments: $d_{T C S}+d_{H C}$ $+d_{M e s}+d_{H C}+d_{T S}$ (see Figure 1(b)). Molecular segments are allowed to have different contributions varying with temperature. Finally, $d$ is written as follows:

$$
d \cong L_{M e s}\left\langle\cos \theta_{M e s}\right\rangle+2 L_{H C}\left\langle\cos \theta_{H C}\right\rangle+2 L_{T S}\left\langle\cos \theta_{T S}\right\rangle .
$$

$L_{M e s}$ is the length of the rigid core, $L_{H C}$ and $L_{T S}$ are the chain lengths both in all trans conformation for alkoxy hydrocarbon linkage and the TS tail, respectively. Each length is defined in Fig. 1. Lengths of molecular segments are calculated from DFT. Different chain conformations are accounted in calculating averages: $\left\langle\cos \theta_{H C}\right\rangle$ and $\left\langle\cos \theta_{T S}\right\rangle$. Results for $\mathrm{C} 4$ and $\mathrm{C} 9$ based on the model are shown in Figure 4. $d(T)$ so calculated agrees with the experimental results from x-ray scattering. Temperature dependence of $d$ is well reproduced using order parameters from IR spectroscopy and lengths from DFT calculations. The main basis of the model therefore is that the layer shrinkage caused by the tilt of the rigid core is balanced by a simultaneous increase in the projection of the length of aliphatic chains along the layer normal. This is termed as: "Compensation model." The given methodology of calculating contributions from each moiety to $d(T)$ has greater potential advantage in designing smectic liquid crystals which exhibit no layer shrinkages in going from SmA down to well within the SmC phase. The basic design involves adjusting lengths of chains in such a way that the rigid sublayer contraction is compensated with an increased projection of the chain length to the layer normal. Molecular tilt angles in SmA observed for $\mathrm{C} 4$ and $\mathrm{C} 9$ shown in Fig. 3 are $\sim 30^{\circ}$ and $\sim 40^{\circ}$, respectively. These provide direct evidence for a large de Vries cone angle in the SmA. A small increase in the molecular tilt angle in SmC is observed. A close agreement between results from the model and experiments is "a direct proof" for de Vries cone model.

Polarized IR spectroscopy enables determination of order parameters of molecular segments. It is shown that the 
aliphatic chains play an important role in determining the layer thickness in smectics. The methodology uses (i) the model independent $\mathrm{x}$-ray scattering results of $d$ and (ii) separate scattering wide angles' peaks observed for the siloxanes and hydrocarbons. The role played by the siloxanes from $\mathrm{X}$-rays ${ }^{4}$ and Raman ${ }^{19}$ is confirmed by IR spectroscopy.

Work of the Dublin and Belfast groups was supported by Award No. 13/US/I2866 from the Science Foundation of Ireland as part of the U.S.-Ireland Research and Development Partnership program jointly administered with the United States National Science Foundation under Grant No. NSF-DMR1410649. The authors (K.M. and A.K.) thank the National Science Centre Poland for Grant No. 2011/03/B/ST3/03369. The authors acknowledge useful discussions with S. Kumar and D. M. Agra-Kooijmann, both from Kent State University, Ohio, USA.

${ }^{1}$ J. P. F. Lagerwall and F. Giesselmann, ChemPhysChem 7, 20 (2006).

${ }^{2}$ G. Pelz, S. Diele, and W. Weissflog, Adv. Mater. 11, 707 (1999).

${ }^{3}$ K. L. Sandhya, Yu. P. Panarin, V. P. Panov, J. K. Vij, and R. Dabrowski, Eur. Phys. J. E 27, 397 (2008).

${ }^{4}$ H. G. Yoon, D. M. Agra-Kooijman, K. Ayub, R. P. Lemieux, and S. Kumar, Phys. Rev. Lett. 106, 087801 (2011).

${ }^{5}$ J. C. Roberts, N. Kapernaum, Q. Song, D. Nonnenmacher, K. Ayub, F. Giesselmann, and R. P. Lemieux, J. Am. Chem. Soc. 132, 364-370 (2010).
${ }^{6}$ A. de Vries, J. Chem. Phys. 71, 25 (1979); Mol. Cryst. Liq. Cryst. 41, 27 (1977); A. de Vries, A. Ekachai, and N. Spielberg, ibid. 49, 143 (1979).

${ }^{7}$ M. V. Gorkunov, F. Giesselmann, J. P. F. Lagerwall, T. J. Sluckin, and M. A. Osipov, Phys. Rev. E 75, 060701R (2007).

${ }^{8}$ M. A. Osipov, M. V. Gorkunov, H. F. Gleeson, and S. Jaradat, Eur. Phys. J. E 26, 395 (2008).

${ }^{9}$ J. P. F. Lagerwall, F. Giesselmann, and M. D. Radcliffe, Phys. Rev. E 66, 031703 (2002).

${ }^{10}$ D. M. Agra-Kooijman, H. G. Yoon, S. Dey, and S. Kumar, Phys. Rev. E 89, 032506 (2014).

${ }^{11}$ See supplementary material at http://dx.doi.org/10.1063/1.4953598 for synthetic details.

${ }^{12}$ A. Kocot and J. K. Vij, Liq. Cryst. 37, 653 (2010); K. Merkel, A. Kocot, J. K. Vij, G. H. Mehl, and T. Meyer, J. Chem. Phys. 121, 5012 (2004).

${ }^{13}$ Yu. P. Panarin, V. Panov, O. E. Kalinovskaya, and J. K. Vij, J. Mater. Chem. 9, 2967 (1999).

${ }^{14}$ N. A. Clark, T. Bellini, R.-F. Shao, D. Coleman, S. Bardon, D. R. Link, J. E. Maclennan, X.-H. Chen, M. D. Wand, D. M. Walba, P. Rudquist, and S. T. Lagerwall, Appl. Phys. Lett. 80, 4097 (2002).

${ }^{15}$ W. Maier and A. Saupe, Z. Naturforsch. A 15, 287-292 (1960).

${ }^{16}$ M. V. Volkenstein, Configurational Statistics of Polymeric Chains (Interscience, New York, 1963).

${ }^{17}$ P. J. Flory, Statistical Mechanics of Chain Molecules (Interscience, New York, 1969).

${ }^{18}$ Y. Shen, L. Wang, R. Shao, T. Gong, C. Zhu, H. Yang, J. E. Maclennan, D. M. Walba, and N. A. Clark, Phys. Rev. E 88, 062504 (2013).

${ }^{19}$ N. Hayashi, T. Kato, A. Fukuda, J. K. Vij, Yu. P. Panarin, J. Naciri, R. Shashidhar, S. Kawada, and S. Kondoh, Phys. Rev. E 71, 041705 (2005). 